\title{
IN SEARCH OF ENTROPY
}

\author{
J. LETESSIER ${ }^{a}$, J. RAFELSKI ${ }^{a, b}$, AND A. TOUNSI ${ }^{a}$ \\ ${ }^{a}$ Laboratoire de Physique Théorique et Hautes Energies*, Universilé Paris 7 \\ Tour 24, 5è ét., 2 Place Jussieu, 75251 Paris CEDEX 05, France \\ ${ }^{b}$ Department of Physics, University of Arizona, Tucson, AZ85721, USA
}

\section{Dedicated to Prof. Iwo Bialynicki-Birula on the occasion of his 60lh birthday}

Entropy is a quantity characterizing the arrow of time in the evolution of a physical system - in every irreversible process the entropy increases. In elementary interactions such as relativistic collisions of two atomic nuclei there is considerable particle production and hence entropy production. We address here a number of questions which arise naturally in this context. When and how is entropy produced in a quantum process, such as is a nuclear collision? How is the particle production related to entropy production? How does one measure the entropy produced in the reaction? We also consider certain fundamental approaches to the problem of entropy definition in quantum physics.

PACS numbers: 25.75.+r, 05.30.Ch, 12.38.Mh

\section{Entropy measurement}

In ultra-high relativistic nuclear collisions performed at energies per nucleon considerably greater than the nucleon rest mass the final state contains a large amount of entropy related to the high particle multiplicity produced, as compared to $\mathrm{N}-\mathrm{N}$ reactions, and to their spectral distributions which are of thermal character. But how does one determine the entropy produced in the elementary collision rcaction? We will present a practical procedure applicable to the environment of relativistic heavy ion collisions and show how one can easily measure the entropy produced. The idea here is to note that the entropy in a thermal gas of particles is well defined, thus all we need to know is how many particles have truly participated in the reaction and how many secondaries they produced. Clearly, the procedure is strongly system and process dependent, and we will therefore provide some necessary details about the physics of the relativistic nuclear collisions. Before proceeding let us quickly recall a few general notions which will allow to pursue this discussion [1-4]:

\footnotetext{
${ }^{*}$ LPTHE is a Unité associée an CNRS.
} 
1) The objective to study complex relativistic interactions of largest nuclei is the search for, and hopefully the discovery of the theoretically predicted new phase of matter in which locally one has deconfinement of colored particles, the so called quark-gluon plasma (QGP). QGP could be formed in such interactions only as a transient state; with time the energy density in the interaction region will decrease due to rapid cooling of this small "sun" and hadronization (conversion into final state hadrons) will ensue - the hadronization of QGP generates certain abundance of hadronic particles, and the transient presence of QGP can be recognized by features such as are unusual abundances of certain particles. For example, in the process of such data analysis we have found that the abundances of strange particles display features which are most easily obtained from hadronizing QGP fireball [1].

2) Aside of the $u, d$ flavors present in projectile and target matter the dense fireball will contain considerable abundance of strange particles, and in general large amount of strangeness production was predicted and experimentally found. The particular use we have for the strange particles, which are most effectively produced in the deconfined QGP phase, is that they can serve to determine the thermal properties of the source: temperature and chemical potentials.

3) In an ensemble of nuclear collisions characterized by a common trigger, the impact parameter can be only determined on average, and the ensemble includes a superposition of different impact parameters and hence different collision systems, which however have common specific properties. Physical observables are therefore best considered in terms that we can relate to the number of directly participating nucleons. Thus the quantity for us to consider here is the entropy produced per baryon participating in the reaction, $\mathcal{S} / \mathcal{B}$, which is also called specific entropy.

4) Often collisions are performed in a frame in which the target nucleus is in the laboratory and hence the center of momentum (CM) frame is moving with considerable rapidity - in the current CERN experiments involving sulphur projectiles at $200 \mathrm{GeV}$ A (per nucleon) the $\mathrm{CM}$ rapidity is 3 for the symmetric collision and about 2.6 for highly asymmetric collisions, in which the effective target consists of a cylinder of matter in the heavy target nucleus with which the lighter projectile overlaps.

5) In order to study the spectral shape of the produced particles, removing the effects associated with the boost of particles from the CM frame to the laboratory frame, one studies the particle yield as function of $m_{\perp}=\sqrt{m^{2}+p_{\perp}^{2}}$ (so called $m_{\perp}$ spectrum) either in a narrow region of rapidity, or with a near $4 \pi$ (global) coverage of all particles.

6) The $m_{\perp}$-spectra of different particles are displaying features which suggest strongly that their source is similar to a thermal heat bath: the spectral shape is close to the Boltzmann exponential and the relative yields can be approximately described by yields of particles in equilibrium (see below) in a state of matter which appears to consist of a mixture of gases in which the species are the different hadrons (pions, nucleons, their resonances, strange hadrons, etc.) - one speaks therefore of a hadronic gas (HG) fireball. 


\subsection{Charged particle mulliplicities and entropy}

Final entropy content of the reaction is most easily visible in the multiplicity of produced particles. We note the relatively high final charged particle multiplicity arising in nuclear collisions: total charged particle multiplicities (excluding target/projectile fragments) above 600 in the central region have been observed by EMU05 (emulsion) cxperiment [5] in $200 \mathrm{GeV} \mathrm{A} \mathrm{S-Pb} \mathrm{collisions,} \mathrm{corresponding}$ to a total final particle multiplicity of up to 1000 . This number does not include non-participating target fragments which are produced in relatively large numbers when the mass of the target considerably exceeds the mass of the projectile as is here the case; these fragments appear primarily in the target frame and are separated from the CM frame participants.

We will show that the ratio of net charge multiplicity to the total charged multiplicity, considered as function of rapidity

$$
D_{Q} \equiv \frac{\frac{\mathrm{d} N^{+}}{\mathrm{d} y}-\frac{\mathrm{d} N^{-}}{\mathrm{d} y}}{\frac{\mathrm{d} N^{+}}{\mathrm{d} y}+\frac{\mathrm{d} N^{-}}{\mathrm{d} y}}
$$

as measured at central rapidity is directly related to the specific entropy $\mathcal{S} / \mathcal{B}[1]$ of the centrally produced highly excited dense matter. The relation between $\mathcal{S} / \mathcal{B}$ and $D_{Q}$ is insensitive to diverse uncertainties inherent in the problem, such as the detailed flavor composition of the observed hadronic system. From diverse detailed studies we conclude that $D_{Q}$ is an extraordinarily valuable experimental variable from which the quantitative information about entropy can be gained, even in the source of hadron radiation is a hadronizing quark-gluon plasma or some other novel form of matter. We will briefly dwell on this matter.

First, we note that in the numerator of $D_{Q}$ the charge of particle pairs produced cancels and it is effectively a measure of the baryon number. Thus in the product $D_{Q}$ with $\mathcal{S} / \mathcal{B}$ the baryon content cancels, and the result is roughly the entropy content of the final state per final state pion. Ilowever, this quantity is insensitive to the structure of the system. In order to be more specific we have performed detailed calculations assuming the equations of state for the source as given by the IIagedorn model of the IIG state [7]. In this model one assumes that the interactions between hadronic constituents are effectively described by considering the diverse hadronic resonances formed in all reactions. Given the equations of state, the value of $D_{Q}$ can be calculated. There are two steps in such a calculation: first one determines the abundances of all hadronic particles as the fireball dissociates for any set of thermal freeze-out parameters. Subsequently, all hadron resonances are allowed to decay into final state stable particles. Similarly we can compute the specific entropy for any set of thermal parameters.

In Fig. 1 we show the product $D_{Q} \cdot(\mathcal{S} / \mathcal{B})$ as a function of $\lambda_{\mathrm{q}}$, the light quark fugacity, for fixed strange quark fugacity $\lambda_{\mathrm{s}}=1 \pm 0.05$ (corresponding to the observed range of values, and characteristic of a decomposing QGP state), with $T$ adjusted at each value of $\lambda_{q}$ to ensure strangeness neutrality - suffice here to say that the range of temperatures arising is compatible with the spectral shape of transverse mass spectra. Another realistic parameter taken here is $\gamma_{\mathrm{s}}=\mathbf{0 . 7}$, thus the strange quark phase space occupancy is $70 \%$. We observe that these curves 


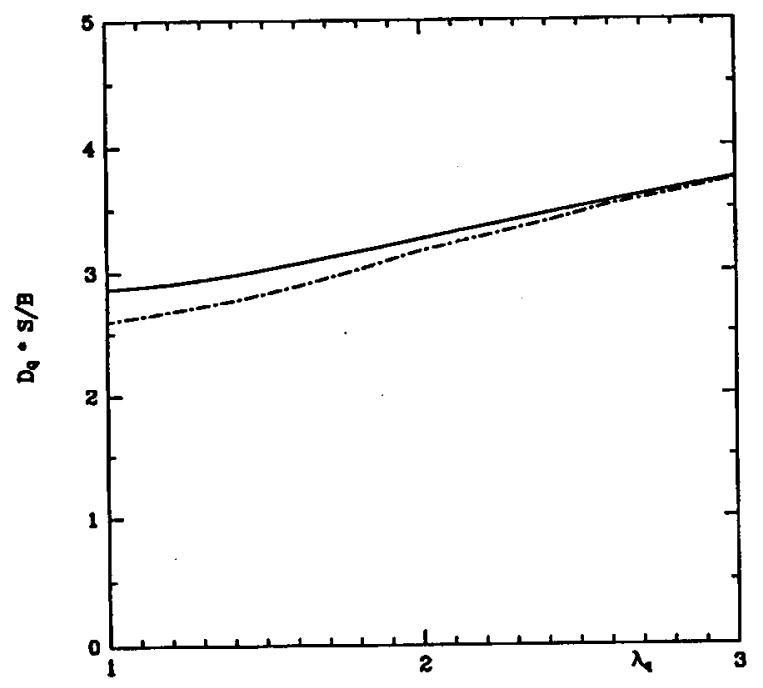

Fig. 1. The product $D_{Q} \cdot(\mathcal{S} / \mathcal{B})$ as function of $\lambda_{q}$ for fixed $\lambda_{s}=1, s-\bar{s}=0$. At each point temperature is adjusted to assure strangeness conservation. The dot-dashed curve was obtained with negligible strange particles content, $\gamma_{s} \rightarrow 0$.

are pretty flat, and the strangeness neutral equilibrium IIG fireball, allowing for all cascading and decays, satisfies the relation (near $\lambda_{q} \approx 1.5$, which applies to current experiments with $200 \mathrm{GeV}$ A sulphur projectiles at CERN)

$$
(\mathcal{S} / \mathcal{B}) \cdot D_{\mathrm{Q}} \approx 3.0 \pm 0.2
$$

determining the entropy content of a central fireball in terms of the normalized charge asymmetry $D_{\mathrm{Q}}$ measured at central rapidity. We note in Fig. 1 a considerable insensitivity of the result to the presence of strange particles: the dash-dotted curves show results obtained with very small fraction of strangeness being present, but with temperature $T$ still constrained by the requirement that for each value of $\lambda_{\mathrm{q}}$ we have strangeness conservation at $\lambda_{\mathrm{s}}=1$.

Experimentally [5] $D_{\mathrm{Q}}=0.085 \pm 0.01$ and hence we find that in collisions of $S$ nuclei with heavy targets at $200 \mathrm{GeV} \Lambda$ the entropy per baryon generated in the collision can be as large as 35 .

\section{Entropy and particle production}

There are many workers today who investigate in a quantitative but phenomenological manner the evolution of a primordial gluon-parton system or more conventional, the evolution of a nucleon and pion gas into the final distributions observed experimentally. Here one views the nuclear collision already as a series of independent collision reactions of diverse (model oriented) projectile and target constituents in which the kinetic energy of the collision is somehow converted into the high particle multiplicity. In such an approach, the determination of (conventionally defined) entropy could in principle be performed at any time during the 
evolution. As soon as one describes the time evolution as a cascade of individual interactions, the reversibility is in principle lost.

We will here show that the period involving particle cascading does not generate much entropy, a finding which did surprise us. Most of entropy was already present after brief initial moments and little entropy is generated while the particles approach their equilibrium abundance, since the production consumes thermal energy of other particles. The result we refer to is pretty elementary and should have been observed long time ago. Our objective now is to show that we have a good definition of entropy in case of a changing number of particles (pair production, Bremsstrahlung, etc.) and to investigate the back reaction arising from the fact that the energy used to make particles needs to be provided by the other particles present. Our findings indicate that most of the cascading evolution towards the equilibrium abundance is just a restructuring in which little entropy is produced, despite a large change in the particle number.

It is useful to appreciate here that the proof of the -licorem found in literature for the relativistic systems is given only for the case of particle number conserving elementary processes [6]. We thus do not know which is the proper definition of entropy $\mathcal{S}=-\mathcal{H}$ which would be an ever increasing object and which would reach its maximum when some equilibrium distribution of particles and their phase space occupancy number is reached. There is, however, a practical way to deal with this problem, without solving first in all generality this probably difficult and intricate issue. The physical systems of interest to us are characterized by relatively large elastic and quasi-elastic cross-sections in which the energy is shared by different particles, which are typically 10-100 times larger than the particle number changing cross-sections. Therefore, it can be assumed that the so called kinetic equilibrium signifying the sharing of the available energy between the available particles, and the establishment of a common temperature, is reached instantaneously as compared to the slowly evolving particle number.

Our strategy then is as follows: for each given particle number we maximize the conventionally defined thermal entropy and in a second step we consider how a change of parlicle number affects this result at fixed energy of the system - in this way we take into account cooling (respectively heating) of the thermal system which yields (respectively absorbs) the energy consumed (respcctively released) in the particle number changing reactions. In order to be able to define properly our nomenclature and to pursue properly the strategy we have indicated, we develop our results from rather elementary first principles.

\subsection{Quantum distributions and entropy}

We recall the discussion of entropy presented by Landau and Lifshitz [8]: from the statistical definition one finds the entropy for each particle species denoted by index $l$ is

$$
\mathcal{S}_{\mathrm{B}, \mathrm{F}}^{l}=\int \mathrm{d} \omega\left[ \pm\left(1 \pm n_{l}\right) \ln \left(1 \pm n_{l}\right)-n_{l} \ln n_{l}\right],
$$

where the integral is over the conventional phase space $\mathrm{d} \omega=\mathrm{d}^{3} p \mathrm{~d}^{3} x /(2 \pi \hbar)^{3}$ (we henceforth choose units such that $\hbar=c=1$ ). The upper + sign applies to the 
Bose particles ('B'), while the lower - sign applies to the Fermi particles ('F'). The Boltzmann limit is applicable when the occupation $n_{l}(x, p)$ of the phase space by the particle species is small compared to unity. We recall

$$
\begin{aligned}
\mathcal{N}^{l} & \equiv \int \mathrm{d} \omega n_{l}, \\
\mathcal{E}^{l} & \equiv \int \mathrm{d} \omega \varepsilon_{l} n_{l},
\end{aligned}
$$

where $\varepsilon_{l}=\sqrt{m_{l}^{2}+p^{2}}$.

The distribution $n_{l}$ follows from the demand that the total entropy of the system $\mathcal{S}=\sum \mathcal{S}^{l}$ be maximal for some given energy of the system $\mathcal{E}=\sum \mathcal{E}^{l}$ and given (for us at any considered time) the number of particles of each species, $\mathcal{N}^{l}$. We find the functional form of $n_{l}(p, x)$ by maximizing the quantity

$$
\mathcal{T}\left[n_{l}\right]=\sum_{l} \int \mathrm{d} \omega\left[ \pm\left(1 \pm n_{l}\right) \ln \left(1 \pm n_{l}\right)-n_{l} \ln n_{l}-\left(\alpha_{l}+\beta \varepsilon_{l}\right) n_{l}\right],
$$

as a functional of $\left\{n_{l}\right\} . \beta=1 / T$ is identified in the usual way as the inverse temperature and $\alpha_{l}$ is related to the fugacity $\gamma_{l}$ :

$$
\gamma_{l}=\mathrm{e}^{-\alpha_{t}},
$$

which permits to prescribe the presence of any given number of particles. The well known functional for the quantum distribution functions follows from this consideration:

$$
n_{\mathrm{B}, \mathrm{F}}=\frac{1}{\mathrm{e}^{\beta \varepsilon+\alpha} \mp 1} .
$$

We reinsert Eq. (7) into the definition of entropy, Eq. (3) and obtain the explicit expressions

$$
\begin{aligned}
& \mathcal{S}_{\mathrm{B}, \mathrm{F}}^{l}=\int \mathrm{d} \omega\left[\frac{\beta \varepsilon_{l}+\alpha_{l}}{\mathrm{e}^{\beta \varepsilon_{l}+\alpha_{l}} \mp 1} \mp \ln \left(1 \mp \mathrm{e}^{-\beta \varepsilon_{l}-\alpha_{l}}\right)\right] \\
& =\int \mathrm{d} \omega\left[\left(\beta \varepsilon_{l}+\alpha_{l}\right) n_{l} \pm \ln \left(1 \pm n_{l}\right)\right], \\
& \mathcal{S}=\frac{\mathcal{E}+P V}{T}+\sum_{l} \alpha_{l} \mathcal{N}^{l} .
\end{aligned}
$$

Note that $\gamma_{l}$ enters as a factor into all Boltzmann exponential $\mathrm{e}^{-\beta \varepsilon}$ and is often therefore referred to as the phase space occupancy factor. All this is text book knowledge, as long as particle number does not change.

\subsection{Conserved quantum numbers}

It is straightforward to introduce constraints, such as are needed when some numbers (e.g. quark number $N_{q}-N_{\bar{q}}$, strangeness, etc.) remain constant, while the numbers of particles change. Physically, this occûrs because quark number changes only by pair production on the time scal . of hadronic interactions. Therefore each quark flavor is separately conserved. This remark incorporates in particular the 
conservation of baryon number and strangeness. Let us denote with $q$ and $\bar{q}$ the distributions of quarks and anti-quarks, respectively. We now should be maximizing the quantity

$$
\begin{aligned}
& \mathcal{T}^{q}[q, \bar{q}]=\int \mathrm{d} \omega[-(1-q) \ln (1-q)-q \ln q-(1-\bar{q}) \ln (1-\bar{q})-\bar{q} \ln \bar{q} \\
& \left.-\alpha_{\mathrm{q}}(q-\bar{q})-\alpha_{\mathrm{p}}(q+\bar{q})-\beta \varepsilon(q+\bar{q})\right],
\end{aligned}
$$

as a functional of $q, \bar{q}$. The Lagrange parameter $\alpha_{q}$ allows to choose a fixed quark number $q-\bar{q}=3 \mathcal{B}$, the parameter $\alpha_{\mathrm{p}}$ allows to choose a given number of $q$ and $\bar{q}$ pairs. The common nomenclature is

$$
\begin{aligned}
\lambda_{\mathrm{q}} & =\mathrm{e}^{-\alpha_{\mathrm{q}}}, \\
\gamma_{\mathrm{q}} & =\mathrm{e}^{-\alpha_{\mathrm{p}}},
\end{aligned}
$$

and $\lambda_{\mathrm{q}}$ is the quark fugacity and $\gamma_{\mathrm{q}}$ is the phase space occupancy parameter. Proceeding as before we find for the distribution functions of quarks and anti-quarks

$$
\begin{aligned}
& q=\frac{1}{\gamma_{q}^{-1} \mathrm{e}^{\beta\left(\varepsilon-\mu_{q}\right)}+1}, \\
& \bar{q}=\frac{1}{\gamma_{q}^{-1} \mathrm{e}^{\beta\left(\varepsilon+\mu_{q}\right)}+1},
\end{aligned}
$$

where we have introduced the (quark) chemical potential

$$
\mu_{\mathrm{q}}=\beta^{-1} \ln \lambda_{\mathrm{q}} \text {. }
$$

\subsection{Equilibrium particle distribution}

As announced we shall now consider what happens in the thermally equilibrated system when we consider the possibility of particle production. First, we shall address the question which values will the Lagrange multipliers $\alpha_{l}$ take at the maximum of entropy, computed at fixed energy $\mathcal{E}$ of the system, which determines the value of $\beta$ (we will presently not include baryon conservation and other constraints, hence the chemical potential $\mu_{\mathrm{q}}=0$ ). Let us consider the energy $\mathcal{E}$ (which does not change) and entropy $\mathcal{S}$ as a function of $\alpha_{l}, \beta$. We have

$$
\mathrm{d} \mathcal{E}=\sum_{l} \frac{\partial \mathcal{E}^{l}}{\partial \alpha_{l}} \mathrm{~d} \alpha_{l}+\frac{\partial \mathcal{E}}{\partial \beta} \mathrm{d} \beta,
$$

which is inserted into the change in entropy to yield

$$
\mathrm{d} \mathcal{S}=\mathrm{d} \mathcal{E} \frac{\partial \mathcal{S} / \partial \beta}{\partial \mathcal{E} / \partial \beta}+\sum_{l} \mathrm{~d} \alpha_{l}\left[\frac{\partial \mathcal{S}^{l}}{\partial \alpha_{l}}-\frac{\partial \mathcal{S}}{\partial \beta} \frac{\partial \mathcal{E}^{l} / \partial \alpha_{l}}{\partial \mathcal{E}^{l} / \partial \beta}\right] .
$$

We first consider the usual case $\mathrm{d} \mathcal{E}=0$. This leaves the last term of Eq. (16), which describes the change of the entropy as the phase space occupancy changes. We introduce

$$
\mathrm{d} \bar{\omega}_{\mathrm{B}, \mathrm{F}}^{l}=\frac{\mathrm{d} \omega}{\left(1 \mp \gamma_{1} \mathrm{e}^{-\beta \varepsilon_{1}}\right)^{2}}
$$


and we obtain

$$
\begin{aligned}
& -\frac{\partial \mathcal{E}}{\partial \beta}=\sum_{l} \int \mathrm{d} \bar{\omega}_{l} \varepsilon_{l}^{2} \gamma_{l} \mathrm{e}^{-\beta \varepsilon_{l}}, \\
& -\frac{\partial \mathcal{E}}{\partial \alpha_{l}}=\int \mathrm{d} \bar{\omega}_{l} \varepsilon_{l} \gamma_{l} \mathrm{e}^{-\beta \varepsilon_{l}}, \\
& -\frac{\partial \mathcal{S}}{\partial \beta}=\sum_{l} \int \mathrm{d} \bar{\omega}_{l} \varepsilon_{l}\left(\beta \varepsilon_{l}+\alpha_{l}\right) \gamma_{l} \mathrm{e}^{-\beta \epsilon_{l}}, \\
& -\frac{\partial \mathcal{S}}{\partial \alpha_{l}}=\int \mathrm{d} \bar{\omega}_{l}\left(\beta \varepsilon_{l}+\alpha_{l}\right) \gamma_{l} \mathrm{e}^{-\beta \varepsilon_{l}} .
\end{aligned}
$$

Considerable cancellation occurs when inserting these relations into Eq. (16), and we find

$$
\left.\mathrm{d} \mathcal{S}^{l}\right|_{E}=\mathrm{d} \gamma_{l} \int \mathrm{d} \bar{\omega}_{l}\left[\alpha_{l} \mathrm{e}^{-\beta \varepsilon_{l}}-\varepsilon_{l} \mathrm{e}^{-\beta \varepsilon_{l}} \frac{\sum_{k} \int \mathrm{d} \bar{\omega}_{k} \alpha_{k} \varepsilon_{k} \gamma_{k} \mathrm{e}^{-\beta \varepsilon_{k}}}{\sum_{k} \int \mathrm{d} \bar{\omega}_{k} \varepsilon_{k} \varepsilon_{k} \gamma_{k} \mathrm{e}^{-\beta \varepsilon_{k}}}\right]
$$

We see that the change in entropy $\mathrm{d} S$ vanishes exactly when all $\alpha_{l}$ vanish (or equivalently, all $\left.\gamma_{l} \rightarrow 1\right)$ ). Furthermore, we can see using Schwartz' inequality that for any $\gamma_{l}<1$ the change $\mathrm{d} S$ is positive for positive $\mathrm{d} \gamma_{l}$ while for $\gamma_{l}>1$ the change is negative, proving that the equilibrium point $\left(\gamma_{l} \rightarrow 1\right)$ is a maximum of entropy at fixed total energy.

It can now be expected considering the laws of thermodynamics that an isolated physical system capable of a self-induced change in particle number will evolve to this equilibrium point, where the entropy at constant energy is maximal. So have we proven the $\mathcal{H}$-theorem? No, since microscopic laws which lead to particle production have to be investigated for each individual situation in order to show that the time evolution of the system is leading to the equilibrium point. In general, for complex physical systems with constraints there may be other maxima of entropy, and moreover, there may be simply no reactions which can lead to the maximum entropy situation.

\subsection{Gluon fireball}

We are now ready to examine the simplest system of dynamical interest to us. We will study for illustrative purposes how a fully equilibrated glue gas state at $T=250 \mathrm{MeV}$ is reached. This (moderately) hot glue ball is a descendent of a still hotter initial glue-parton ball which was once far from particle abundance equilibrium and in which glue interactions have been producing particles at the same time as the temperature needed to drop as the fixed energy was shared by ever larger number of constitucnts. Our expectation and belief when we started this exercise was that a lot of entropy is produced as the system develops towards the particle abundance equilibrium. However, we shall show now that this is indeed not so! The reason is that as the equilibrium in particle number abundance is approached, we must adjust the temperature of the system, and the result of a very subtle balance between the different effects is that we find below considerable constancy of the entropy of the system.

We see this most easily considering the Boltzmann approximation: the factor $\gamma$ becomes a normalization factor which describes the average occupancy of the 
phase space relative to the equilibrium value, the additive term describes how the entropy per particle changes as the occupancy changes. We find in Boltzmann limit

$$
\begin{aligned}
& \mathcal{N}^{l}=\left.\gamma_{l} \mathcal{N}^{l}\right|_{\text {eq }}, \\
& \mathcal{E}^{l}=\left.\gamma_{l} \mathcal{E}^{l}\right|_{\text {eq }}, \\
& \mathcal{S}^{l}=\left.\gamma_{l} \mathcal{S}^{l}\right|_{\text {eq }}+\left.\ln \left(\gamma^{-1}\right) \gamma_{l} \mathcal{N}^{l}\right|_{\text {eq. }} .
\end{aligned}
$$

For massless particles the phase space integral is easily performed and one obtains

$$
\begin{aligned}
& \mathcal{N}^{0}=a V \gamma_{l} T^{3}, \\
& \mathcal{E}^{0}=3 a V \gamma_{l} T^{4}, \\
& \mathcal{S}^{0}=4 a V \gamma_{l} T^{3}+\ln \left(\gamma^{-1}\right) a V \gamma_{l} T^{3},
\end{aligned}
$$

where $a=g_{l} / \pi^{2}$ and $g_{l}$ is the degeneracy. One easily finds how for $\mathcal{E}^{0}=$ const the entropy as function of $\gamma$ varies

$$
\mathcal{S} \propto \gamma^{\frac{1}{4}}(4-\ln \gamma) .
$$

This functional has a very weak maximum at $\gamma=1$. For example we note that at $\gamma=2$ the entropy is at $98.3 \%$ of the value at $\gamma=1$.

At this point it is perhaps prudent to review the more familiar case of a fixed temperature environment and to briefly consider a thermally insulated box in which the energy loss due to radiation is externally compensated keeping the temperature constant, as in e.g. the usual black body radiator. The radiation spectrum displays the Bose shape, which minimizes the free energy $\mathcal{F}\left(\gamma_{l}, T\right)$ content of the gas at fixed temperature. Recalling now that $\mathcal{F}=\mathcal{E}-T \mathcal{S}$ we can combine Eqs. (20)-(22) which gives in the Boltzmann limit

$$
\mathcal{F}^{l}=-a V T^{4} \gamma_{l}\left[1+\ln \left(\gamma_{l}^{-1}\right)\right],
$$

which naturally has the minimum at $\gamma_{l}=1$. However, one now finds that a change by factor two in $\gamma$, at fixed $\beta$ leads to a change by $35 \%$ in the value of the free energy and even a greater change in entropy. Thus we see that at fixed $\beta$ the equilibrium point $\gamma_{l} \rightarrow 1$ is much better defined, than at fixed $\mathcal{E}$.

We now return to consider the isolated system at fixed $\mathcal{E}$. The situation is much less trivial when we look at what happens when we consider the more general situation of a quantum gas at finite mass. We performed a numerical calculation for a system of gluons. Our calculations are set up such that the particle abundance equilibrium results at $T=0.250 \mathrm{GeV}$ - due to energy conservation and constancy of volume the energy density is always constant as function of $\gamma_{\mathrm{G}}$ (index ' $\mathrm{G}$ ' is henceforth dropped) and takes the value $2.66 \mathrm{GeV} / \mathrm{fm}^{3}$. We will also consider a thermal mass for gluons $m_{\mathrm{G}}^{\text {th }}=0.200 \mathrm{GeV}$, in which case we also choose to have equilibrium at $T=0.250 \mathrm{GeV}$ and therefore the energy density drops to $1.89 \mathrm{GeV} / \mathrm{fm}^{3}$.

We show in Fig. 2 the entropy density $\mathcal{S} / V$ (units $1 / \mathrm{fm}^{3}$ ) as function of $\gamma$. We note that for massless gluons we cannot proceed beyond $\gamma=1$ because of Bose-condensation phenomena not considered further here. We show by dashed lines the same calculation performed using as glue mass the thermal value $m_{\mathrm{G}}^{\text {th }}=$ $0.200 \mathrm{GeV}$, which allows us to continue the calculation up to $\gamma \approx 2.7$. The remarkable feature to be noted in Fig. 2 is the appearance of the very weak maximum at 
$\gamma=1$. However, the temperature $T$ shown in Fig. 3 evolves rapidly in the interval $0.1<\gamma<1$.

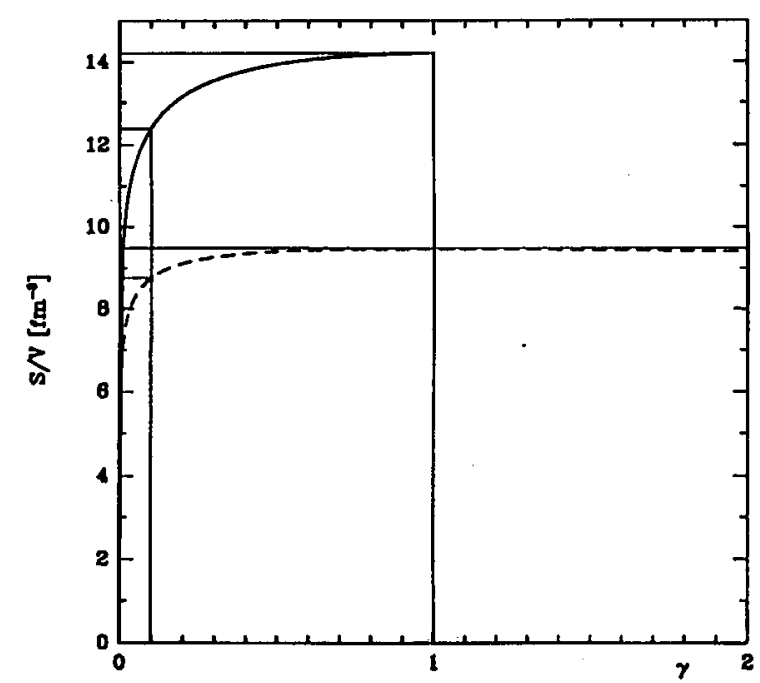

Fig. 2. Entropy density $S / V$ (units $1 / \mathrm{fm}^{3}$ ) at fixed energy density $E / V=$ $2.66 \mathrm{GeV} / \mathrm{fm}^{3}$ for $m_{\mathrm{G}}=0$ (solid line) and at $E / V=1.89 \mathrm{GeV} / \mathrm{fm}^{3}$ for $m_{\mathrm{G}}=0.200 \mathrm{GeV}$ (dashed line) for a (gluon) Bose gas as function of the chemical occupancy $\gamma$.

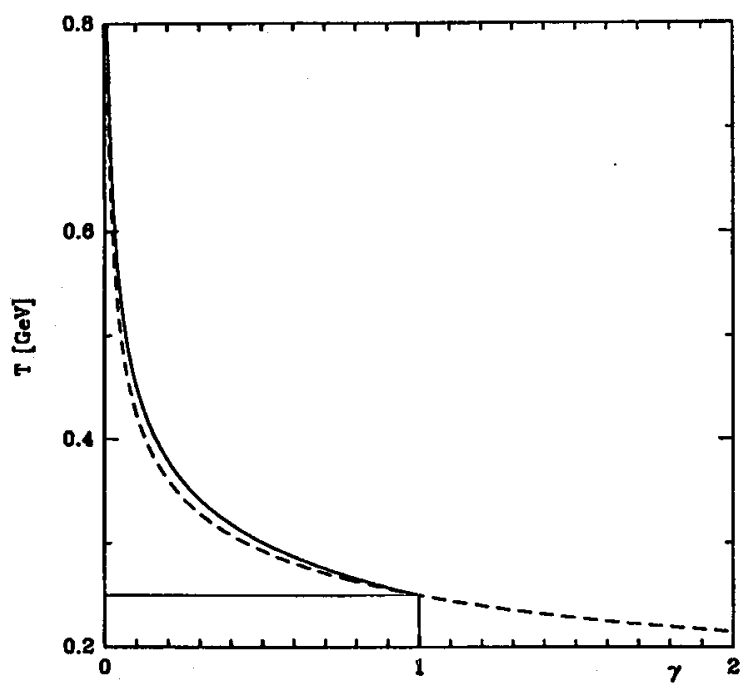

Fig. 3. The temperature $T$ as function of the chemical occupancy $\gamma$. Lines as in Fig. 2; equilibrium point $\gamma=1$ has been chosen to occur at $T=0.250 \mathrm{GeV}$. 


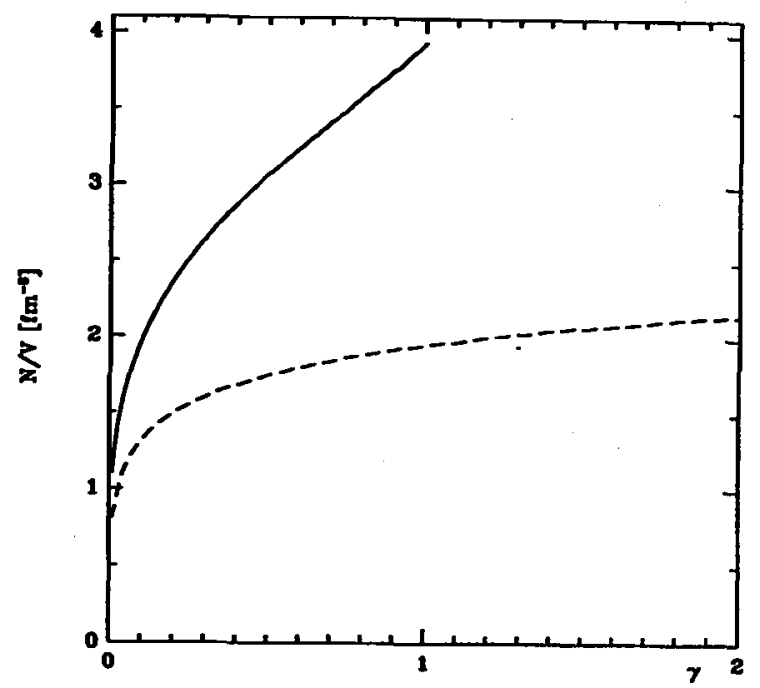

Fig. 4. Particle density $N / V$ (units $1 / \mathrm{fm}^{3}$ ) as function of the chemical occupancy $\gamma$. Lines as in Fig. 2.

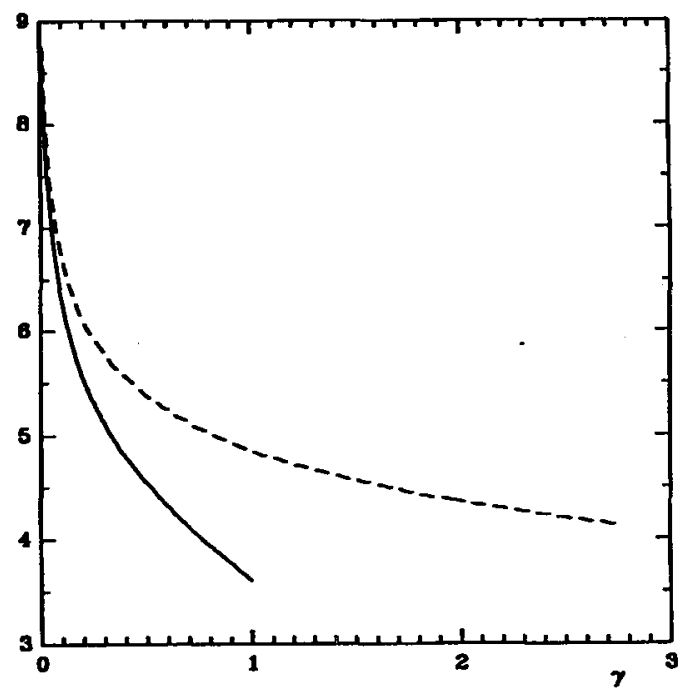

Fig. 5. Entropy per particle $S / N$ for a (gluon) Bose gas as function of the chemical occupancy $\gamma$. Lines as in Fig. 2.

As we can further note in Fig. 4 the glue number density increases considerably in the same region - it more than doubles for massless gluons. In Fig. 4 we also note that the glue number density increases monotonically as we pass $\gamma=1$ 
for $m_{\mathrm{G}}>0$. On the other hand, Fig. 5 shows that the entropy per particle drops continuously - all these results are as expected from the qualitative result obtained in the Boltzmann limit above, though we now see the remarkable flatness of the massive Bose gas case beyond $\gamma=1$. This may be interpreted as a new liberty of such a gas to over-saturate the phase space under certain constraints, which could be experimentally noticed in the central rapidity spectra of pions produced in the nuclear reactions at low encrgies, due to the resulting distortion of the boson spectral shape.

\subsection{Expanding volume}

A more realistic model of the nuclear reaction requires inclusion of other particles and consideration of the volume expansion and obviously this is not the place to develop this further. However, we note that in the expanding quark-glue fireball there are no other known sources of entropy, since the expansion is assumed to be a largely entropy conserving hydrodynamical flow process. This quasi entropy conserving evolution is confirmed within the model of the parton cascade [4] which also displays the unusually rapid production of the entropy in the initial first instant of the collision. To do a similarly quantitative calculation we need in principle to know with considerable precision the relative rates of change of the volume $V$ and the occupancy factor $\gamma$, the former driven by quantum transport theory, the latter presumably by the microscopic processes, which are in principle also part of a transport formulation (see below).

Details of even a phenomenological discussion are by necessity model dependent, but we can obtain some interesting qualitative insights combining in qualitative terms the expansion dynamics with the particle production processes and cooling discussed above. For a (nearly) massless Bose gas we have

$$
\begin{aligned}
& \mathcal{S}=4 a_{1} V T^{3} \gamma+\ln \left(\gamma^{-1}\right) a_{2} V T^{3} \gamma, \\
& \mathcal{E}_{\text {th }}=\mathcal{E}_{0}-\mathcal{E}_{\text {flow }}=3 a_{1} V T^{4} \gamma,
\end{aligned}
$$

where $\mathcal{E}_{0}$ is the initial energy from which the flow term is subtracted. Both $a_{1}(\gamma, \beta m)$, and $a_{2}(\gamma, \beta m)$ (which reduce to $a$ in the Boltzmann limit) are easily determined factors and can be exactly computed. We find in particular for $\beta m \rightarrow 0$ :

$$
\frac{a_{1}}{a_{2}}=\frac{\sum_{n=1}^{\infty} \frac{\gamma^{n-1}}{n^{4}}}{\sum_{n=1}^{\infty} \frac{\gamma^{n-1}}{n^{3}}}=\left\{\begin{array}{r}
0.9 \text { for } \gamma=1, \\
1 \text { for } \gamma=0 .
\end{array}\right.
$$

In the Boltzmann limit, $a_{1}$ and $a_{2} \rightarrow a$, and both are independent of $\alpha=\ln \gamma^{-1}$; we find the example discussed earlier. We therefore take $a_{1} / a_{2} \approx 1$. From Eq. (28) we obtain

$$
\mathcal{S} \approx S_{0} \frac{T_{0}}{T} \frac{1+\alpha / 4}{1+\alpha_{0} / 4}\left(1-\frac{\mathcal{E}_{\text {flow }}}{\mathcal{E}_{0}}\right) .
$$

We see in Eq. (30) that the impact of the flow, which reduces the reservoir of thermal energy is to reduce the growth in entropy due to particle production. Since entropy increase was rather small anyway, it is now possible to suppose that 
we have $S / S_{0} \approx 1$. Then we find from Eq. (30) that the temperature drop (or rise) on the way to particle equilibrium is given by

$$
\frac{T_{\mathrm{H}}}{T_{0}} \approx \frac{1}{1+\alpha_{0} / 4}\left(1-\frac{\mathcal{E}_{\mathrm{H}}}{\mathcal{E}_{0}}\right)
$$

where $T_{\mathbf{H}}$ is the final temperature with full particle abundance equilibrium. We see that both hydrodynamical expansion and particle production cooling combine to increase the temperature reduction, while both do not lead to appreciable entropy increase.

\section{Entropy production in elementary interactions}

We have seen now how the final state allows us to determine the entropy production, and further, we have to our surprise discovered that this entropy was most likely produced in a very early stage of the nuclear collision when the hot, nearly thermal distribution of partons was generated. This find hag, seems at present completely inescapable and hence the issue becomes to formulate a theoretical framework in which we can describe the initial quantum evolution and particle formation. It is evident that a framework for such an approach can only be found in a general quantum transport theory based e.g. on a generalization of the Wigner distribution function [9]. Bialynicki-Birula and collaborators [10] have formulated just a suitable theoretical framework in order to tackle this difficult problem, and we shall recall briefly its essential elements before proceeding.

\subsection{Relativistic Wigner function}

In Wigner's formulation of quantum phase space distribution, the function $W(x, p ; t)$ which is the analog of the classical phase space distribution function, is a split-point Fourier transform of the quantum density matrix. For the relativistic quantum matter field $\Psi$ it has been shown [10] that the proper gauge invariant, relativistic generalization of the Wigner function can be

$$
\begin{aligned}
& W_{i, j}(r, p, t)=-\frac{1}{2} \int \mathrm{d}^{3} s \mathrm{e}^{-\mathrm{i} p \cdot s} \\
\times & \left\langle\Phi\left|\exp \left[-\mathrm{i} e \int_{-1 / 2}^{1 / 2} \mathrm{~d} l s \cdot \boldsymbol{A}(\boldsymbol{r}+l \hbar s)\right]\left[\Psi_{i}(\boldsymbol{r}+\hbar s / 2, t), \Psi_{j}^{\dagger}(r-\hbar s / 2, t)\right]\right| \Phi\right\rangle,
\end{aligned}
$$

where the spinor indices $i, j=0, \ldots, 3$. The coordinate $r$ and momentum $p$ are the phase space variables of the state $|\Phi\rangle$. Both $\boldsymbol{r}$ and $\boldsymbol{p}$ are similar to classical phase space variables and do not contain $\hbar$, which has been shown explicitly above. The vector potential $\boldsymbol{A}$, chosen in the Heisenberg-Pauli (temporal) gauge, is in principle composed of two contributions: the external sources which do not undergo the dynamic evolution here discussed and are assumed to be smooth in (microscopic) world, and the so called "back reaction" terms, arising from the induced matter field source current, which include the highly oscillating part (Zitterbewegung) of a frequency $\nu \sim 2 m c^{2} / \hbar$ with a mean distance $\lambda_{\mathrm{e}} \sim \hbar / m c$ (the Compton wavelength). 
In order to see the physical limits of the formalism the gauge invariant and relativistic quantum Wigner function, Eq. (32), of a spin 1/2-matter field is decomposed into 16 distribution functions, amenable to direct physical interpretation,

$$
W=\frac{1}{4}\left(f_{0}+\sum_{i=1}^{3} \rho_{i} f_{i}+\sigma \cdot g_{0}+\sum_{i=1}^{3} \rho_{i} \sigma \cdot g_{i}\right),
$$

where $\rho_{i}, \sigma_{i}$ and $\rho_{i} \sigma_{j}$ are $4 \times 4$ matrices. The distribution functions $f_{i}(x, p, t)$ and $g_{i}(x, p, t)$ have precise physical meaning which can be inferred from their momentum integrals: $\left(f_{0}, g_{1}\right)$ form the current four vector phase space distribution, $f_{3}$ is the mass distribution, $g_{0}$ is the spin distribution, $g_{3}$ is the magnetic moment distribution, etc. We do not have here the space to discuss in any detail the complex dynamics that the relativistic quantum distribution functions $f_{i}, g_{i}$ satisfy. These are first order differential equations in $\partial_{t}, \nabla$ and nonlocal (or infinite order) in $\partial_{p}$ of the 16 real functions $\left(f_{i}, g_{i}\right)$. It is the non-locality in $p$ which reflects on the intrinsically quantum character of the distributions: In the time variable there appears to be complete reversibility in this complex dynamical problem, as we should expect in quantum theory.

\subsection{Classical limil and loss of reversibility}

The full time evolution of this system contains both quantum flow phenomena and particle production. It is totally reversible even if we recall that the field $\boldsymbol{A}$ comprises the back reaction arising from the produced particles - but only if this back reaction itself arises in a time-reversible way. This may be a tricky issue since as is customary in quantum field theory both advanced and retarded potentials are to be incorporated at quantum level, but only retarded potentials are implemented at classical level. We leave for now this issue which has been in the literature for some 50 years [12].

One can actually show [13] that the dynamical equations possess in a $\hbar \rightarrow 0$ expansion the good dynamical limit yielding in particular for both particles and anti-particles the so called Vlasov equations, which describe the reversible flow of a given particle and anti-particle assembly in presence of electromagnetic fields. The variables of the problem which show this interesting limit are particle and anti-particle phase space distributions, defined such that the number of particles and anti-particles remains equal if we begin the dynamic evolution in a vacuum state

$$
f_{ \pm}^{c}(p)= \pm \frac{1}{2}\left[f_{0}^{c}( \pm p) \pm \frac{E_{p}}{m c^{2}} f_{3}^{c}( \pm p)\right] .
$$

In the limit $\hbar \rightarrow 0$ we arrive at [13]:

$$
0=\partial_{t} f_{ \pm}^{c}+v \cdot \nabla f_{ \pm}^{c} \pm e\left(\boldsymbol{E}+\frac{1}{c} v \times \boldsymbol{B}\right) \cdot \partial_{p} f_{ \pm}^{c}
$$

An interesting point to observe here is that the two Vlasov equations, which emerge naturally in the classical limit, are definitively not entropy generating, and that they also do not contain the process of primordial particle production, indeed this process did not occur in an expansion around $\hbar \rightarrow 0$. 
So how do we find and identify in fundamental transport theory the primordial particle production (and presumably also in some sense entropy generating) processes? How do we establish in a fundamental manner dynamical equations in generalized semiclassical limit, where the flow phenomena are described in the $\hbar \rightarrow 0$ limit, while the particle production phenomena are retained? With regard to these questions the formulation of the quantum transport theory in the physical phase space [10] has a definitive advantage over other equivalent approaches [11]. While the problem is not fully solved today, an important step in this direction was made when the fundamental modes of the BGR-Wigner function [10] were determined: it is possible to recast the dynamical equations which the relativistic quantum Wigner distribution satisfies into the form of an integral equation, in which the kernel is a propagator function, and the singularities of its Fourier transform are characterizing the fundamental modes. We refer the reader for details to the work of Białynicki-Birula et al. [14]. Here we note that these singularities comprise both flow and pair modes: the pole structure of the (retarded) propagator is

$$
-\mathrm{i} \tilde{G}_{\mathrm{ret}}^{0}=\frac{\left(\omega+\boldsymbol{L}_{0}[q]\right)\left[\omega^{2}-\left(\omega_{+}^{2}+\omega_{-}^{2}-L_{0}^{2}[q]\right)\right]}{\left.\left[(\omega+\mathrm{i} \varepsilon)^{2}-\omega_{+}^{2}\right]\left[(\omega+\mathrm{i} \varepsilon)^{2}-\omega_{-}^{2}\right)\right]} .
$$

The causality condition on $G_{\text {ret }}^{0}$ is guaranteed by the standard device of prescribing pole positions via introducing $\varepsilon \rightarrow 0^{+}$. IIere $\widetilde{G}_{\text {ret }}^{0}(q, p)$ is the Fourier transformed $\boldsymbol{r} \rightarrow \boldsymbol{q}$ kernel $G_{\mathrm{ret}}^{0}(\boldsymbol{r}, p)$, and $\boldsymbol{L}_{0}[q]$ is the Fourier transform of a suitable linear differential operator acting in conventional phase space. The retarded character of the matter propagator in the phase space is not breaking the time reversal invariance, indeed it assures it since the time reversed solutions are simply generated looking at backward propagation with an advanced kernel. The choice of the retarded boundary condition enforces causality on the action of matter fields. We also note that the here chosen boundary condition of the matter field does lead to the standard perturbative expansion in terms of the Feynman diagrams (which comprise advanced and retarded solutions, at least to first order in the interaction, as was shown [14] by explicit commutation of the vacuum polarization function. The poles of the kernel are at

$$
\omega_{ \pm}=\frac{1}{\hbar}\left(E_{p+q / 2} \pm E_{p-q / 2}\right)
$$

given in terms of energies

$$
E_{\boldsymbol{p} \pm \boldsymbol{q} / 2}=\sqrt{m^{2}+(\boldsymbol{p} \pm \boldsymbol{q} / 2)^{2}}
$$

The upper sign in Eq. (37) leads to singularities with a minimal energy of $2 m$, corresponding to pair modes, while the lower sign leads to singularities for $\boldsymbol{p} \cdot \boldsymbol{q} \rightarrow 0$ and thus corresponds to flow modes. Only these modes are found in the classical limit. In the full theory, both dynamical modes are appearing next to each other in full equivalence, however the presence of the threshold $2 m$ in the pair case tells that only when these high frequencies are present in the system we should expect to excite pairs in the vacuum, and that the classical limit in which these pair modes appear must be found in very judicious approach, since we cannot take simply the limit $\hbar \rightarrow 0$, nor can we coarse grain (that is average in the phase space), 
as the wavelengths associated with particle production are at the scale of the Compton wavelength. A first principle solution to this problem is still not found; phenomenologically one can proceed simply to add suitable particle (and entropy!) source terms to the generalized Vlasov equations [15]. In such a phenomenological description, reversibility is lost.

We see here that indeed a general framework to study the formation of the initial entropy rich state has been formulated - we have a quantum transport theory which generates naturally the classical motion as a limit $\hbar \rightarrow 0$ and which is capable to treat the nonperturbative aspects of particle production. However, there is so far no understanding of the subtle transition from the full reversible quantum theory to the classical limit accompanied by formation of particles and entropy.

\section{In search for entropy}

We complete our study by returning to consider the fundamental problem when and how was the entropy observed experimentally produced. We found that the production must have occurred early on in the collision process, when the system was most probably subject to the laws of quantum physics - one usually imagines that in this period the evolution ought to be time reversal invariant, thus entropy conserving, until we somehow reach to the classical, asymptotic limit. But are quantum evolution processes really truly reversible when particle production is abundant? Quantum systems also evolve in time, and though in principle they remain reversible, it can be easily argued that this reversibility becomes a mathematical finesse e.g. soon after many particle pairs are produced into a large volume. The separating (due to momentum conservation) particle pairs cannot continue for ever to remain in exact phase coherence which will permit them to find each other in order to re-annihilate, this remark is particularly true at high energies here considered. Thus the Liouville time (time to recover initial state) grows beyond the measure of infinity, on the scale of the life time of the Universe and in particular, any slightest perturbation of the system will destroy the time reversibility. Thus once even the slightest observation of the system is done, this latent entropy hidden in the particles produced, becomes physically present. The objective then appears to be to find a definition of entropy which sees the virtual appearance of entropy in quantum reactions, such that there is no discontinuity associated with the time growth of entropy. These remarks show clearly that one of the important steps is to find the suitable definition which will be practicable in the quantum domain.

The central problem is that there is presently no useful definition of entropy in the quantum domain: we recall the definition originally considered by von Neumann in terms of density matrix $\rho$ :

$$
\mathcal{N}=-\operatorname{Tr} \rho \ln \rho,
$$

This proposed form has an appearance which strongly resembles the usual Boltzmann expression. Unfortunately, this is a very misleading semblance since $\mathcal{N}$ is a constant of motion (as are traces of any function of $\rho$ satisfying the dynamics 
$\mathrm{d} \rho / \mathrm{d} t=\mathrm{i}[\rho, H])$, and hence this definition implies that the entropy does not grow in the quantum domain. Were this to be strictly true, all entropy produced in nuclear collisions would appear in a discontinuous way at the instance the quantum evolution is observed experimentally. Such a description of reality is not palatable and therefore we believe that a more useful definition ought to be found - the interplay of the flow and particle production phenomena as we described earlier in a quantum transport theory provides an intriguing point of departure in such a search for a better definition.

Is the entropy an intrinsically classical concept or is there a more general form of the quantum entropy definition, as we have been suggesting, which will allow to introduce an effective arrow of time already at the quantum level? We strongly believe in the latter, more rational conclusion, and further also see a close relationship with the presence of inelastic reactions, in our discussion of the particle production type, more generally any chemical, atomic, nuclear reaction, with which is associated an effectively irreversible change of the structure of the constituents.

In summary, we have described an effective way to measure entropy produced in relativistic nuclear collisions, we have studied the entropy evolution of an isolated fireball at fixed energy and have shown that while it approaches the particle number equilibrium, there is very little entropy produced. We took this to imply that entropy was already generated at early stages in the collision, when the dynamics obeyed quantum physics, but we could not determine how it was produced - and we took this observation as another stimulus to seek entropy production in elementary quantum transport theories.

\section{Acknowledgments}

Portions of this work were performed in collaboration with: I. Bialynicki-Birula, E.D. Davis, P. Górnicki, U. Heinz, G.R. Shin and J. Sollfrank.

The work of J.R. was in part supported by DOE, grant no. DE-FG02$-92 \mathrm{ER} 40733$.

\section{References}

[1] J. Letessier, A. Tounsi, U. Heinz, J. Sollfrank, J. Rafelski, Paris preprint PAR/LPTHE/92-27 (revised) submitted to Phys. Rev. D.

[2] J. Letessier, J. Rafelski, A. Tounsi, submitted to Phys. Rev. Lett., Preprint PAR/LPTHE/93-51.

[3] J. Letessier, A. Tounsi, U. Heinz, J. Sollfrank, J. Rafelski, Phys. Rev. Lett. 70, 3530 (1993).

[4] K. Geiger, Phys. Rev. D 46, 4986 (1992).

[5] Y. Takahashi, EMU05 Collab., private communication and to be published.

This result is presented in more detail in: J. Rafelski, J. Letessier, A. Tounsi, Proc. XXVI Int. Conf. on IIigh Energy Physics, Dallas (1992), Amer. Inst. of Physics Proceedings No. 272, 1993, and in Ref. [1].

[6] S.R. de Groot, W.A. van Leeuwen, Ch.G. van Weert, Relativistic Finetic Theory, North-Holland, Amsterdam 1980. 
[7] R. Hagedorn, Cargèse Leclures in Physics, Vol. 6, Gordon and Breach, New York 1973.

[8] L. Landau, E. Lifshitz, Statistical Physics, Pergamon Press, London 1959, Sec. 40, 54.

[9] E. Wigner, Phys. Rev. 40, 749 (1932).

[10] I. Białynicki-Birula, P. Górnicki, J. Rafelski, Phys. Rev. D 44, 1825 (1991).

[11] H.-Th. Elze, U. Heinz, Phys. Rep. 183, 81 (1989).

[12] J.A. Wheeler, R.P. Feynman, Rev. Mod. Phys. 21, 425 (1949).

[13] G.R. Shin, J. Rafelski, Phys. Rev. A 48, 1869 (1993).

[11] I. Białynicki-Birula, E.D. Davis, J. Rafelski, Phys. Lett. B 311, 329 (1993).

[15] Y. Kluger, J.M. Eisenberg, B. Svetitsky, F. Cooper, E. Mottola, Phys. Rev. Lett. 67, 2427 (1991). 